\title{
Tattoo Based Identification: Sketch to Image Matching
}

\author{
Hu Han and Anil K. Jain \\ Department of Computer Science and Engineering \\ Michigan State University, East Lansing, MI 48824, U.S.A. \\ $\{$ hhan, jain\}@msu.edu
}

\begin{abstract}
Tattoos on human body provide important clue to the identity of a suspect. While a tattoo is not an unique identifier, it narrows down the list of identities for the suspect. For these reasons, law enforcement agencies have been collecting tattoo images of the suspects at the time of booking. A few successful attempts have been made to design an automatic system to search a tattoo database to identify near-duplicate images of a query tattoo image. However, in many scenarios, the surveillance image of the crime scene is not available, so the query is in the form of a sketch of a tattoo (as opposed to an image of a tattoo) drawn based on the description provided by an eyewitness. In this paper, we extend the capability of tattoo image-to-image matching by proposing a method to match tattoo sketches to tattoo images using local invariant features. Specifically, tattoo shape is first extracted from both tattoo sketch and tattoo image using Canny edge detector. Local patterns are then extracted from tattoo shape as well as tattoo image (appearance) using SIFT. A local feature based sparse representation classification scheme is then used for matching. Experimental results on matching 100 tattoo sketches against a gallery set with 10,100 tattoo images show that the proposed method achieves significant improvement (rank-200 accuracy of 57\%) compared to a state-of-the-art tattoo image-to-image matching system (rank-200 accuracy of 19\%).
\end{abstract}

\section{Introduction}

Soft biometric traits, e.g. scars, marks, and tattoos (collectively called SMT) are being increasingly used to complement primary biometric identification systems based on fingerprint, face, or iris [14]. In fact, criminal investigations have leveraged soft biometric traits as far back as the late 19 th century $[6,25]$. For example, the first personal identification system, the Bertillon system, tried to provide a precise and scientific method to identify criminals by using physical measurements of body parts, especially measure-
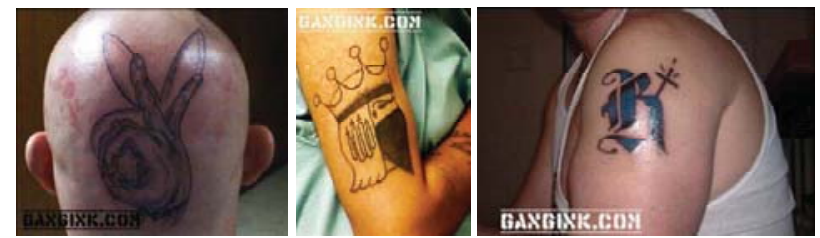

Figure 1. Some examples of gang tattoos ${ }^{1}$

ments of the head and face, as well as recording individual scars, marks, and tattoos on the body. Due to the importance of soft biometric traits, the US Federal Bureau of Investigation (FBI) is developing the Next Generation Identification (NGI) system [28] for identifying criminals, where palm print, face, iris, and SMT will be used to augment fingerprint evidence.

Among various soft biometric traits, tattoos, in particular, have received substantial attention over the past several years due to their prevalence among the criminal section of the population and their saliency in visual attention (See Fig. 1). Tattoos have been used as a sign by individuals to distinguish themselves from others for thousands of years [1]. A recent survey by The Harris Poll shows that there has been a huge increase in popularity of tattoos among U.S. adults; about one in five U.S. adults have at least one tattoo $(21 \%)$ which is up from $16 \%$ when the same survey was conducted in 2003 [2].

In forensic investigations and law enforcement scenarios, tattoos engraved on the human body have been successfully used to assist in human identification [16]. Tattoo pigments are embedded in the skin to such a depth that even severe skin burns often do not destroy a tattoo. For this reason, tattoos were found to be useful in identifying victims of the 9/11 terror attacks in 2001 and the Asian tsunami in 2004 [5]. Criminal identification is another important application of tattoos because tattoos often contain hidden information related to a suspect's criminal history (e.g., gang membership, previous convictions, years spent in jail, etc.).

\footnotetext{
${ }^{1}$ http: //www $\cdot$ gangink. com/index $\cdot$ php?pr=GANG_LIST
} 


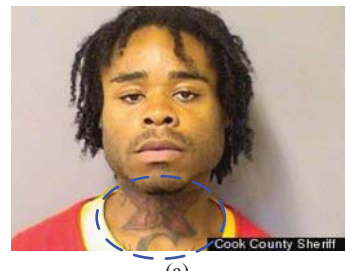

(a)

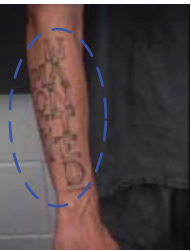

(b)

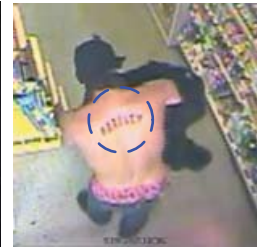

(c)
Figure 2. Tattoos assist in arrest of suspects. (a) A murder suspect was caught following the detailed description of the red, fivepointed star tattoo on his neck ${ }^{2}$. (b) A black ink "Most Wanted" tattoo in block letters running down the right forearm lead to arrest of a suspect of a bank robbery ${ }^{3}$. (c) Tattoo captured in a surveillance video lead detectives to the arrest of a man who broke into the Pit Stop gas station in Monkey Junction ${ }^{4}$.

Tattoos are particularly useful when any primary biometric trait like face or fingerprint is not available. Figs. 2 (a, b, c) show three cases reported in the media where suspects were successfully identified and apprehended based on tattoos on their body.

Despite the growing use of tattoos in law enforcement agencies, there has been only a limited amount of research on automatic tattoo matching. The prevailing practice of tattoo matching relies on keyword-based matching. For example, law enforcement agencies usually follow the ANSI/NIST-ITL1-2011 standard ${ }^{5}$ for assigning a single keyword to a tattoo image in the database. However, a keyword-based tattoo image retrieval has several limitations in practice [20]: (i) The ANSI/NIST classes define a limited vocabulary which is insufficient for describing various tattoo patterns; (ii) multiple keywords may be needed to adequately describe a tattoo image; (iii) human annotation is subjective and different subjects can give dramatically different labels to the same tattoo.

To overcome the limitations of keyword-based tattoo matching, Jain et al. [16] proposed a content-based image retrieval (CBIR) system, called Tattoo-ID to perform image-to-image tattoo matching. Tattoo-ID extracts keypoints from tattoo images using scale invariant feature transform (SIFT) [22] and uses an unsupervised ensemble ranking algorithm [19] to measure the visual similarity between two tatto images. Acton and Rossi [3] proposed to extract global features (e.g., edge direction and color) from

\footnotetext{
2http://www.huffingtonpost.com/2012/08/02/ demonte-williams-murder-s_n_1734237.html

${ }^{3}$ http: //www.tampabay.com/news/publicsafety/ crime/article1249486.ece

${ }^{4}$ http://portcitydaily.com/2012/09/26/ tattoos-aid-in-arrest/

${ }^{5}$ The ANSI/NIST-ITL1-2011 standard defines eight major classes (human, animal, plant, flag, object, abstract, symbol, and other) and a total of 70 subclasses (including male face, cat, narcotics, American flag, fire, figure, national symbols, and wording) for categorizing tattoos.
}

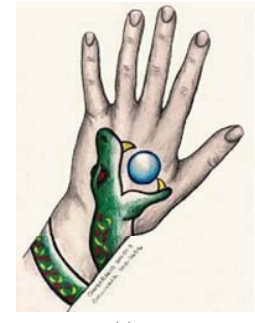

(a)

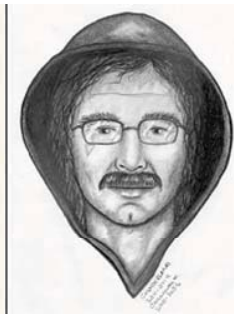

(b)
Figure 3. Tattoo and face sketches of a suspect who attempted an abduction of a 13-year-old girl. These sketches were released by the Royal Canadian Mounted Police (RCMP) in Chilliwack in January $2011^{6}$. The suspect was described as (a) having a blue and green tattoo of a snake on the back of his left hand, and (b) a 30year Caucasian male five feet six inches tall with a slim build.

tattoo images using active contour segmentation and skin detection. Lee et al. [20] improved the performance of the Tattoo-ID system by developing a more robust similarity measures and incorporating the metadata associated with tattoo images. Heflin et al. [13] proposed a method to detect and classify scars, marks and tattoos under unconstrained conditions, and adapted Tattoo-ID system to a scenario of open set classification.

The above CBIR systems were designed to solve imageto-image tattoo matching problem, which assumes that the query tattoo is available as an image. These systems further assume that the query image is a "near duplicate" of the true tattoo image if present in the database. However, in many cases, the tattoo image of a suspect may not be available (e.g., scenarios without surveillance cameras). In these circumstances, just like a face sketch [18], a sketch of a tattoo can be drawn based on the description provided by an eyewitness or the victim. Fig. 3 shows a publicly released tattoo sketch, along with a face sketch of a suspect by the Royal Canadian Mounted Police (RCMP), which were drawn following the verbal description from an eyewitness. In case where the suspect is wearing a face mask, the face sketch cannot be drawn, and tattoo sketch may become the main clue for identifying the suspect. For these reasons, there is a need for developing automatic tattoo sketch to image matching methods. While face sketch recognition has received some attention in the face recognition community $[10,18,27,30,32,33]$, to our knowledge, no work has been reported on tattoo sketch to tattoo image matching.

There are two main challenges in automatic tattoo sketch to image matching: (i) Tattoo sketch to image matching is a cross modality matching problem, where the texture and color of the sketch and image can be quite different; (ii) The eyewitness may not always be able to provide an ac-

\footnotetext{
${ }^{6}$ http: //www.huffingtonpost. com/2012/08/02/ demonte-williams-murder-s_n_1734237.html
} 

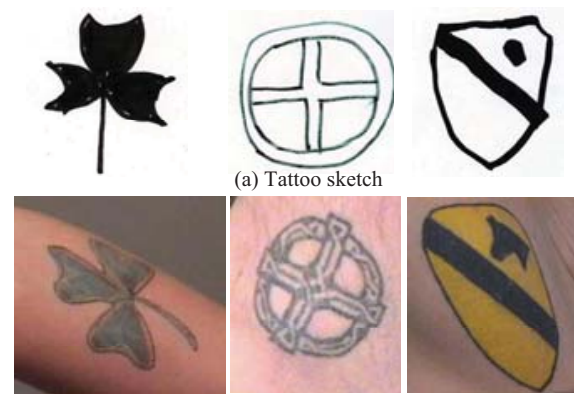

(b) Tattoo image

Figure 4. Exemplar tattoo sketches and their corresponding tattoo images ${ }^{7}$.

curate description of a suspect's tattoo, leading to a possibly non-linear deformation between a tattoo sketch and the corresponding tattoo image. Further, there may be a significant loss in the detail of tattoo sketches. Several tattoo sketches and their corresponding tattoo images are shown in Figure 4, which illustrate the above challenges.

In this paper, we design and build a prototype of an automatic tattoo sketch to image matching system. The objectives of this work are to (i) construct a tattoo sketch database for studying the tattoo sketch to image matching problem, (ii) provide a common representation for tattoo sketch and image that can suppress intra-class variations while maintaining inter-class discriminative ability, (iii) leverage local invariant features to represent tattoos, and (iv) effectively match tattoo sketches against a large tattoo image gallery.

\section{Tattoo Sketch Database}

There is no operational tattoo sketch data set that we could find from law enforcement agencies. So, in our study, we construct a data set consisting of 100 tattoo sketches drawn by two different subjects, each sketch corresponding to a known tattoo image. The protocol for drawing the tattoo sketch is illustrated in Fig. 5. A tattoo image was first shown to a subject for one minute. Ten minutes later, the subject was asked to draw a tattoo sketch (a line drawing image) on a white paper according to his/her memory. The tattoo viewing time and the time gap between viewing the tattoo and drawing the sketch were selected for expediency purposes. The tattoo sketches drawn on the paper were then digitized with a scanner. Examples of tattoo sketches and their corresponding tattoo images are shown in Figs. 4 and $5^{8}$. In addition to these 100 tattoo sketch and image pairs, we also made use of a data set of 10,000 tattoo images provided by the Michigan State Police to populate the gallery.

\footnotetext{
${ }^{7}$ The tattoo images were provided by the Michigan State Police.

${ }^{8}$ The 100 tattoo sketch and image pairs used in this work are available to interested researcher through our lab's website: http://www.cse.msu.edu/biometrics/pubs/databases.html
}

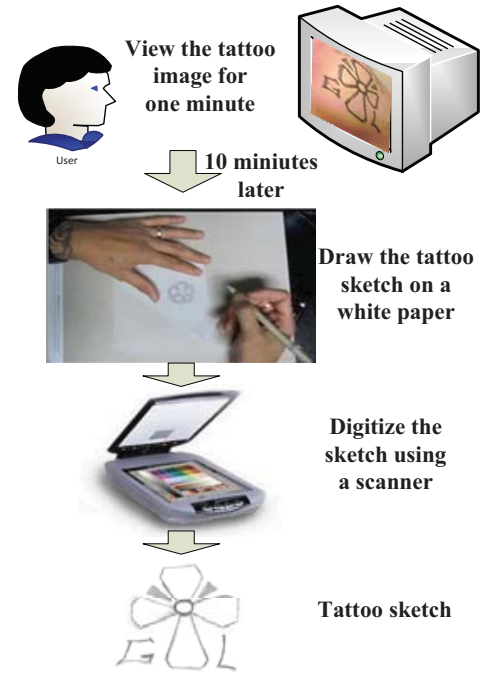

Figure 5. An illustration of the procedure used to construct the tattoo sketch database used in this study.

\section{Sketch to Image Matching}

In many object recognition tasks, alignment is the key step. For example, in face recognition, two eyes are commonly used to normalize face images. However, since different faces have the same geometry, face alignment can leverage this property during landmark detection and alignment. By contrast, objects in tattoo images can be of arbitrary shape, which makes it difficult to establish the correspondence.

For the tattoo sketch to image matching task, there are additional challenges, namely the modality difference and deformation between the two entities to be matched. This suggests the use of local feature similarity in matching a tattoo sketch to a tattoo image. Specifically, it would be desirable to determine whether there exist some local patterns or structures that appear in both the sketch and the image. As illustrated in Fig. 6, the proposed approach first extracts the tattoo shapes from both the sketch and the image using an edge detector. Local patterns are then detected from the edge map (tattoo shape) using the SIFT operator [22]. Finally, local pattern based sparse representation classifier (SRC) $[21,31]$ is utilized to measure the similarity between a tattoo sketch and a tattoo image.

\subsection{Tattoo shape extraction}

Tattoo images which are captured using digital cameras, usually contain a significant amount of texture information (See Fig. 4). However, detailed texture can hardly be depicted in hand drawn tattoo sketches. A tattoo sketch drawn based on verbal description provided by a witness (Fig. 5) mainly describes the shape of the tattoo. This is understandable because studies in human vision suggest that "sim- 


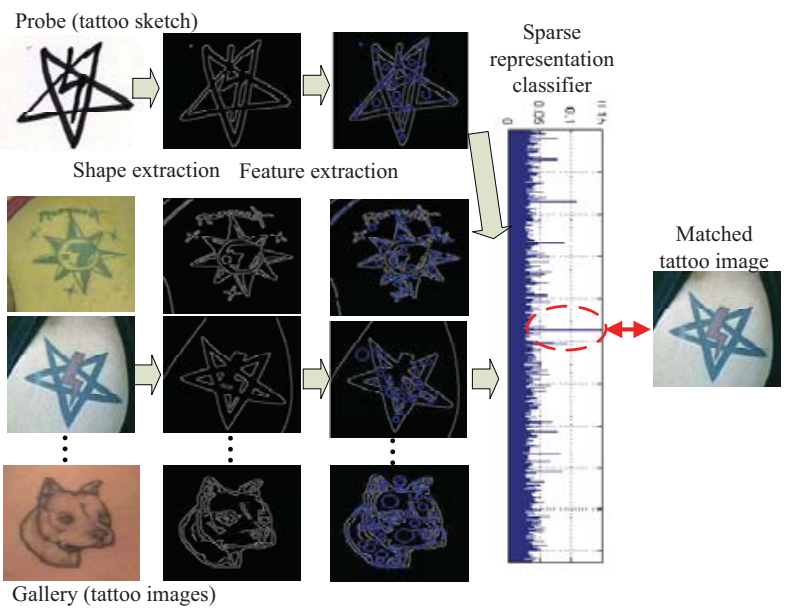

Figure 6. Overview of the proposed approach for matching a tattoo sketch to database of tattoo images.

ple cells" in striate cortex are responsible for edge detection, and are fairly sensitive to sharp changes in intensity [24]. Following this observation, we propose to match tattoo sketches to tattoo images by focusing on the matching of shape (structure) information ${ }^{9}$.

Deformable templates have been defined to detect the shape of a particular class of objects. For example, Active Shape Models [9] have been widely used for the shape detection of faces, hands, etc. However, tattoos can be of arbitrary shape, which makes it prohibitive to predefine deformable templates. Instead of representing the shape of an arbitrary tattoo using deformable templates, we directly use the edge map extracted by the Canny edge detector [8] to describe the tattoo shape ${ }^{10}$. In our experiments involving Canny edge detector, we used a $7 \times 7$ Gaussian filter with $\sigma^{2}=2$ for image smoothing, and set the value of $T$ in the range $[0.14,0.35]$ for hysteresis thresholding.

Fig. 7 shows shape information extracted from some of the tattoo sketches and images. As shown in Fig. 7 (a), for tattoo images or sketches with high contrast, the extracted tattoo shape information provides a good representation. The extracted shape emphasizes the tattoo structure, and deemphasizes the skin texture differences between tattoo sketch and tattoo image. Additionally, the extracted shape reduces the modality gap between the sketch and the image, which simplifies the feature representation step.

We also observed that for tattoo images or sketches with low contrast (e.g. Fig. 7 (b)), the extracted shape informa-

\footnotetext{
${ }^{9}$ In this work, tattoo shape is not limited to just describing the external boundary of a tattoo, but also the internal structure.

${ }^{10}$ We also tried some other edge detection methods reviewed in [26], e.g. gradient edge detectors like Sobel and Prewitt, Laplacian of Gaussian (LoG), etc., and found Canny detector to be the best in our tattoo sketch to image matching experiments.
}
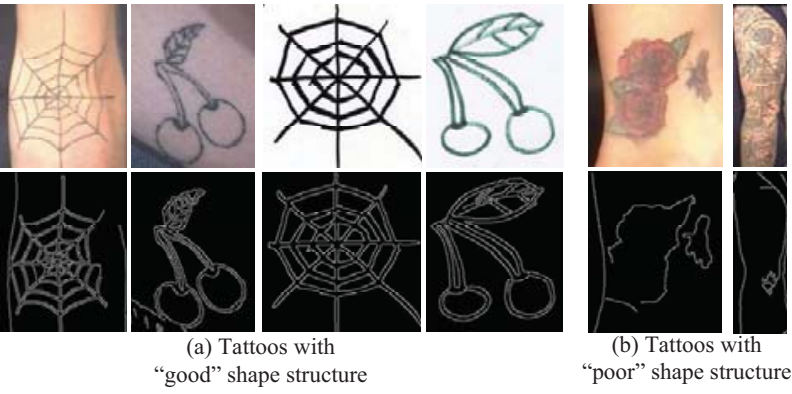

Figure 7. Tattoo shape extraction using Canny edge detector. (a) Tattoos with well defined shape structure; (b) Tattoos with poorly defined shape structure

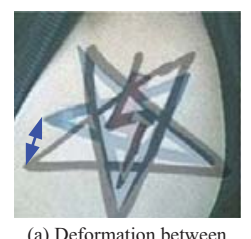

(a) Deformation between holistic patterns

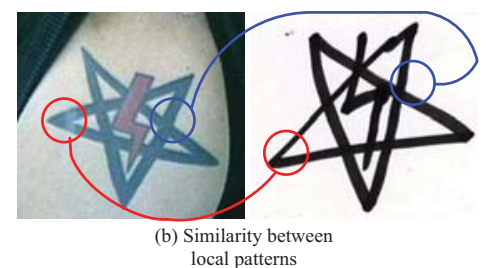

local patterns
Figure 8. Feature representation approaches: holistic vs. local ${ }^{12}$. (a) Holistic variations between tattoo image and sketch due to deformation and geometric transform; (b) Similarity between tattoo image and sketch based on local patterns.

tion is poor and a significant portion of the structure information contained in the tattoo is $\operatorname{los}^{11}$. In the next section, we will present a complementary method for handling tattoos with poor shape information.

\subsection{Feature representation}

As mentioned in Section 3, because of the presence of tattoo shape deformation between tattoo sketch and image, it is challenging to establish a correspondence between their holistic shapes. We further illustrate this challenge in Fig. 8 (a) by overlapping a tattoo sketch with its corresponding tattoo image. By contrast, if we look at the tattoo sketch and the corresponding tattoo image in local neighborhoods, we find that there are relatively minor deformations between them (See the local patterns illustrated with red and blue circles in Fig. 8 (b)). In this section, we propose a method to represent individual tattoo shapes based on their local patterns.

Local pattern based methods have found considerable success in a variety of computer vision applications, like object recognition [22], image retrieval [17, 29], and image mosaicing [7]. The SIFT detector proposed by Lowe [22] is probably the most widely used local operator. SIFT provides a description of an object in an image by detecting

\footnotetext{
${ }^{11}$ In our future work, we also plan to utilize image preprocessing methods $[11,12]$ to enhance the tattoo patterns.
} 


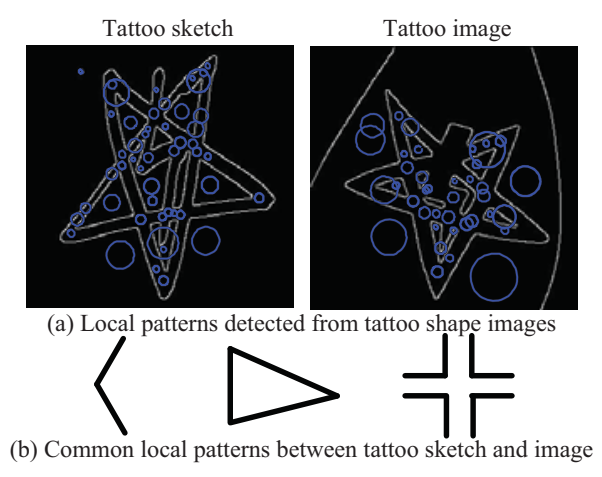

Figure 9. Salient local patterns detected from tattoo shapes. (a) Shapes of tattoo sketch and tattoo image; (b) Some of the common local patterns detected between tattoo sketch and tattoo image.

salient image regions (interesting points, or keypoints). The salient image regions usually lie in high-contrast regions of the image, for example object edges, such that they can be repeatedly detected under changes in viewpoint that induces translation, rotation, and scaling of image, as well as noise and illumination variations.

Unlike most other computer vision applications where SIFT is applied to detect keypoints in gray-scale images, we utilize SIFT to detect local patterns from tattoo shape images. Fig. 9 (a) shows the salient local patterns detected from tattoo shapes using SIFT. Fig. 9 (b) illustrates some of the common local patterns that are detected in both tattoo sketch and image. These common local patterns characterize the similarity between tattoo sketch and image.

Some of the tattoo shapes extracted from the tattoo image are not very informative due to low image quality (See Fig. 7 (b)). As a result, only a few salient local patterns can be detected from such tattoo shapes (See Fig. 10 (b)). To resolve this problem, for each tattoo sketch or tattoo image, we also detect salient local patterns in the tattoo appearance image (See Fig. 10 (c)). The salient local patterns detected from tattoo shape image and tattoo appearance image are separately used for matching, and the two scores are summed together to get the final matching score between a tattoo sketch and a tattoo image.

The deformation between a tattoo sketch and image is greatly reduced w.r.t. each common local pattern; the remaining variations are mainly due to translation, rotation, and scaling, which are easily handled by various descriptors, such as SIFT [22], SURF [4], SAAI [23]. In this work, we used a $4 \times 4$ SIFT descriptor with 8 orientations $\left(0^{\circ}\right.$, $45^{\circ}, 90^{\circ}, 135^{\circ}, 180^{\circ}, 215^{\circ}, 270^{\circ}$, and $\left.315^{\circ}\right)$ to represent each local pattern, resulting in a feature vector with $128 \mathrm{di}-$ mensions.

\footnotetext{
${ }^{12}$ To show the overlapped tattoos more clearly, we did not use the shape information detected in Section 3.1.
}

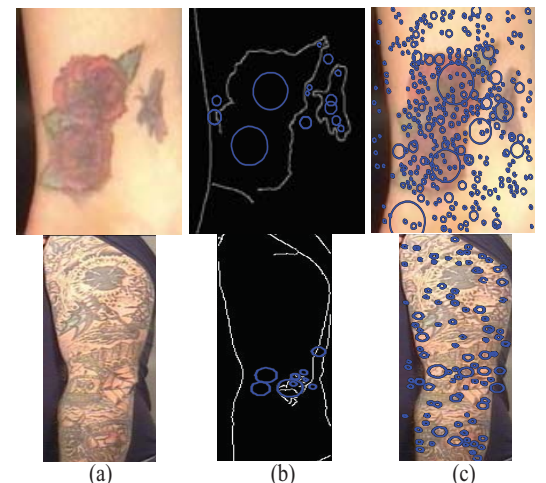

Figure 10. Local pattern detection in two different tattoo images. (a) Tattoo images; (b) local patterns detected from the tattoo shape image; (c) local patterns detected from the appearance image.

\subsection{Matching}

Sparse Representation-based Classification (SRC) has been found to be very effective for face recognition [21, 31]. It was also reported in [21, 31] that a block or keypoint based SRC classifier is even more robust than SRC based on holistic gray scale images. Inspired by this idea, we propose to match tattoo sketch to tattoo image using a local pattern based SRC. Specifically, for a probe tattoo sketch, we denote its local pattern features as $Y=\left\{\boldsymbol{y}_{i} \mid i=1,2, \cdots, m\right\}$, where $m$ is the number of local patterns, and $\boldsymbol{y}_{i}$ is a 128dimensional feature vector. A sparse representation for each local pattern $\boldsymbol{y}_{i}$ can be formulated as

$$
\hat{\boldsymbol{x}}_{i}=\arg \min \left\|\boldsymbol{x}_{i}\right\|_{1} \text { s.t. } \boldsymbol{y}_{i}=\boldsymbol{A} \boldsymbol{x}_{i},
$$

where $\boldsymbol{x}_{i}$ is a sparse coefficient vector, $\|\cdot\|_{1}$ is the $\ell^{1}$ norm of a vector, and $A$ is the gallery dictionary, which is the concatenation of all features for local patterns detected from $k$ tattoo images in the gallery set ${ }^{13}$

$$
\begin{aligned}
\boldsymbol{A}= & {\left[\boldsymbol{A}_{1}, \boldsymbol{A}_{2}, \cdots, \boldsymbol{A}_{k}\right] } \\
= & {\left[\boldsymbol{a}_{1,1}, \boldsymbol{a}_{1,2}, \cdots, \boldsymbol{a}_{1, n_{1}} ; \boldsymbol{a}_{2,1}, \boldsymbol{a}_{2,2}, \cdots, \boldsymbol{a}_{2, n_{2}} ; \cdots ;\right.} \\
& \left.\boldsymbol{a}_{n, 1}, \boldsymbol{a}_{n, 2}, \cdots, \boldsymbol{a}_{k, n_{k}}\right] .
\end{aligned}
$$

We then classify $\boldsymbol{y}_{i}$ by assigning it to the tattoo class $\hat{j}$ that can represent $\boldsymbol{y}_{i}$ with the minimum reconstruction error

$$
\begin{aligned}
\hat{j} & =\arg \min _{j} e_{j}\left(\boldsymbol{y}_{i}\right) \\
& =\arg \min _{j}\left\|\boldsymbol{y}_{i}-\boldsymbol{A} \delta_{j}\left(\hat{\boldsymbol{x}}_{i}\right)\right\|_{2},
\end{aligned}
$$

where $e_{j}(\cdot)$ is the reconstruction error for $y_{i}$ by using local patterns in tattoo class $j, \delta_{j}(\cdot)$ is a function which only selects coefficients corresponding to tattoo class $j$ from $\hat{\boldsymbol{x}}_{i}$, and $\|\cdot\|_{2}$ is the $\ell^{2}$ norm of a vector.

\footnotetext{
${ }^{13}$ In our gallery set with 10,100 tattoo images, each tattoo image is viewed as a separate class.
} 
Since there are totally $m$ salient local patterns detected from the probe tattoo sketch, the larger the number of common local patterns between tattoo sketch and tattoo image, the higher their similarity. Based on (3), we can formulate the classification of a probe tattoo sketch as

$$
\begin{aligned}
\hat{j} & =\arg \min _{j} \sum_{i=1}^{m} e_{j}\left(\boldsymbol{y}_{i}\right) \\
& =\arg \min _{j} \sum_{i=1}^{m}\left\|\boldsymbol{y}_{i}-\boldsymbol{A} \delta_{j}\left(\hat{\boldsymbol{x}}_{i}\right)\right\|_{2},
\end{aligned}
$$

Finally, the local patterns detected from tattoo appearance images are also taken into consideration for tattoo images with poor shape information

$$
\hat{j}=\arg \min _{j}\left(\sum_{i=1}^{m} e_{j}^{S}\left(\boldsymbol{y}_{i}\right)+\sum_{i=1}^{m} e_{j}^{A}\left(\boldsymbol{y}_{i}\right)\right),
$$

where $e_{j}^{S}(\cdot)$ and $e_{j}^{A}(\cdot)$ are, respectively, the reconstruction errors for $y_{i}$ by using local patterns detected from tattoo shape image and appearance image.

\section{Experimental Results}

\subsection{Evaluation metrics}

Forensic scenarios with sketch matching (tattoo or face) generally involve an examination by the eyewitness or detective of the top few hundred retrieved tattoo images ${ }^{14}$. Hence, the proposed tattoo sketch to image matching method is evaluated by examining the top-200 retrieval rate using a Cumulative Match Characteristic (CMC) curve. The accuracy of the proposed method is compared against a state-of-the-art image-to-image tattoo matching system, Tattoo-ID [16, 20].

\subsection{Robustness to deformations}

In this section, we evaluate the robustness of the proposed tattoo sketch to image matching approach against several common types of deformation. We consider three types of deformations, i.e., rotation, shear-warp, and twirl (See Fig. 11). Fig. 12 shows two tattoo sketches (A and B) that are first deformed, and then matched to the gallery tattoo images.

Fig. 12 (a) shows the matching scores and retrieval ranks for the two tattoo sketches with and without manual deformations. For the rotated tattoo sketches, the matching scores range from 0.55 to 1.0 , and the retrieval ranks range from rank-1 to rank-22, which demonstrates that the proposed approach is fairly robust against rotation ${ }^{15}$. Viewpoint changes lead to shear-warp deformations between a

\footnotetext{
${ }^{14}$ This kind of examination is routinely practiced by law enforcement agencies [15] even for latent fingerprint matching.

${ }^{15}$ Left or right $90^{\circ}$ rotation is exactly one of the 8 orientations of the SIFT descriptor. That is why the matching scores for tattoo sketches with $90^{\circ}$ rotations are as high as the original tattoo sketch.
}

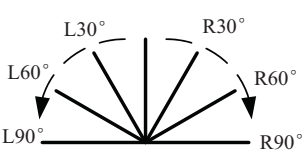

(a) Rotation

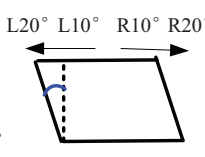

(b) Shear

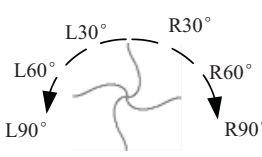

(c) Twirl
Figure 11. Three types of deformation for probe tattoo sketch. (a) Clockwise and counterclockwise rotations; (b) Left and right shear-warps; and (c) Clockwise and counterclockwise twirls.

tattoo sketch and a tattoo image. Fig. 12 (b) shows that the proposed approach is robust to shear-warp deformations ${ }^{16}$. Compared with rotation and shear-warp, twirl is a more challenging deformation for tattoo sketch to image matching (See the tattoo sketches in the bottom row of Fig. 12 (c)). However, the proposed approach can still match most (10 out of 12) of the deformed tattoo sketches within top200 rank. These experiments reveal the effectiveness of local invariant features for tattoo representation.

\subsection{Matching performance}

We evaluate the performance of the proposed approach by matching 100 tattoo sketches to 10,100 tattoo images. A state-of-the-art image-to-image tattoo matcher, called Tattoo-ID [20] is used as the baseline. As shown in Fig. 13, the matching rates of Tattoo-ID at rank-100 and rank-200 are $13 \%$ and $19 \%$, respectively. The performance of TattooID demonstrates the difficulty of tattoo sketch-to-image matching. By contrast, the proposed system achieves significantly higher matching rates than Tattoo-ID. For example, the matching rates of our system at rank-100 and rank-200 are $48 \%$ and $57 \%$, respectively ${ }^{17}$.

Fig. 14 shows some examples of good and poor matchings by the proposed approach. Fig. 14 (a) shows three good matches where rank-1 is the correct match. We can find that even in the presence of deformations, the matching score is high. We also noticed that while two tattoos may have several common local patterns, their global structures can be completely different (See the second example in Fig. 14 (b)). This suggests the exploration of structural constraints to further improve our method.

\section{Summary}

Tattoos on human body provide valuable clue to establish the identity of a suspect or a victim of a crime. While tattoo images are routinely collected by law enforcement

\footnotetext{
${ }^{16}$ Even though the R20 shear-warp for tattoo sketch A leads to a high matching rank (e.g. rank-199), this is still a promising match w.r.t. our rank-200 evaluation metric.

${ }^{17} \mathrm{On}$ a Windows platform with an Intel Core 2 2.4G processor and 4GB RAM, the average retrieval times of Tattoo-ID (C++ implementation) and the proposed approach (Matlab implementation) are 30 and 50 seconds, respectively, for a gallery of size 10,100 .
} 


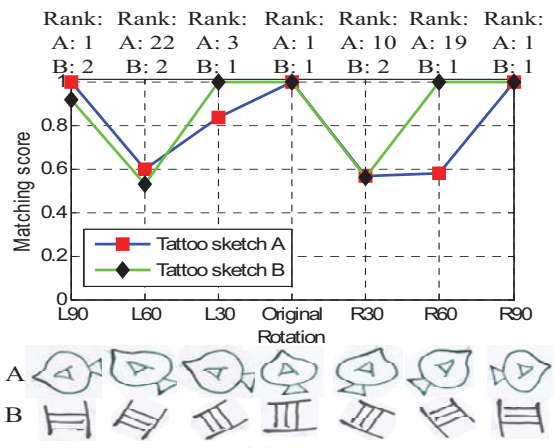

(a)

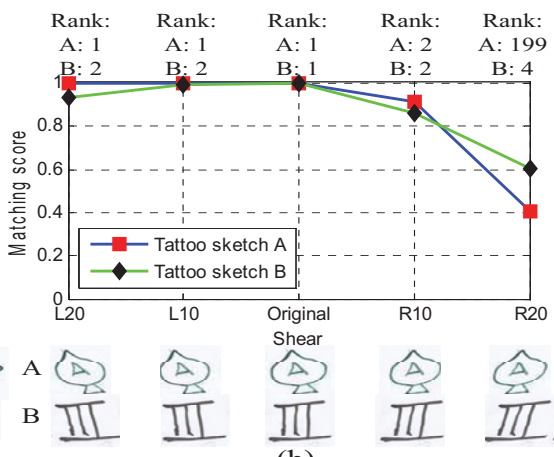

(b)

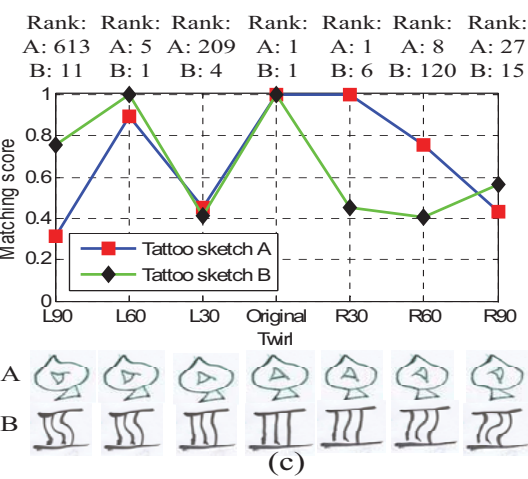

Figure 12. Robustness of the proposed approach against three types of deformation for two exemplar tattoo sketches (A and B): (a) rotation, (b) shear-warp, and (c) twirl.

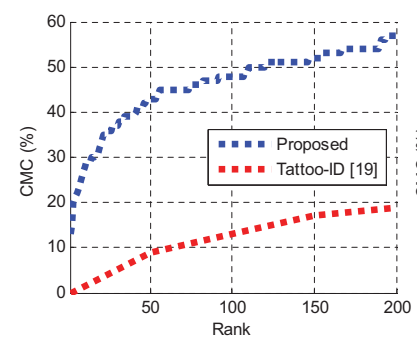

(a) Overall performance

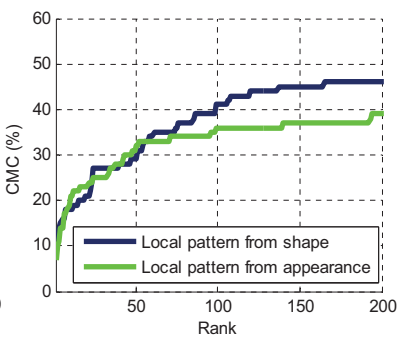

(b) Local pattern from shape vs. appearance

Figure 13. Matching performance of the proposed approach for tattoo sketch to image matching. (a) Comparisons with a stateof-the-art tattoo image-to-image matcher, Tattoo-ID [20]. (b) Performance of local patterns detected from tattoo shape image and tattoo appearance image.

agencies, their use so far has been limited due to lack of automatic tattoo matching systems. Recent work on automatic tattoo matching, for instance $[16,20]$, has shown the ability to identify near-duplicate tattoos. We have extended the state-of-the-art in tattoo matching by devising a method to match tattoo sketch to tatoo image. We constructed a tattoo sketch database with 100 tattoo sketches, and proposed a scheme to match tattoo sketches to tattoo images using local invariant features. The proposed approach was found to be robust against deformations like rotation, shear-warp, and twirl. Our method significantly outperforms a state-ofthe-art image-to-image tattoo matcher.

In our future work, we plan to enlarge the tattoo sketch database by using the Amazon Mechanical Turk (AMT) crowdsourcing service. We also would like to improve our approach by integrating human annotations of tattoos, and by introducing structural constraints between various local patterns extracted in both tattoo image and sketch. The differences between tattoo image-to-image matching and sketch-to-image matching will also be further investigated.

\section{Acknowledgement}

This research was supported by grants from the NSF Center for Identification Technology Research (CITeR).

\section{References}

[1] A brief history of tattoos. http://www. designboom. com/history/tattoo_history.html.

[2] One in five U.S. adults now has a tattoo. http://www.harrisinteractive.com/

NewsRoom/HarrisPolls/tabid/447/mid/1508/ articleId/970/ctl/ReadCustomo20Default/ Default.aspx.

[3] S. Acton and A. Rossi. Matching and retrieval of tattoo images: Active contour cbir and glocal image features. In Proc. IEEE SSIAI, pages 21-24, 2008.

[4] H. Bay, A. Ess, T. Tuytelaars, and L. V. Gool. Speededup robust features (SURF). Comput. Vis. Image Underst., 110(3):346-359, 2008.

[5] J. P. Beauthier, P. Lefevre, and E. D. Valck. Autopsy and identification techniques. The Tsunami Threat-Research and Technology, N. A. Mörner, 2011.

[6] A. Bertillon. Signaletic Instructions Including the Theory and Practice of Anthropometrical Identification. The Werner Company, 1896.

[7] M. Brown and D. Lowe. Recognising panoramas. In Proc. ICCV, pages 1218-1225, 2003.

[8] J. Canny. A computational approach to edge detection. IEEE Trans. Pattern Anal. Mach. Intell., 8(6):679-698, 1986.

[9] T. Cootes, C. Taylor, D. Cooper, and J. Graham. Active shape models-their training and application. Comput. Vis. Image Underst., 61(1):38-59, 1995.

[10] H. Han, B. Klare, K. Bonnen, and A. K. Jain. Matching composite sketches to face photos: A component-based approach. IEEE Trans. Inf. Foren. Sec., 8(1):191-204, 2013.

[11] H. Han, S. Shan, X. Chen, and W. Gao. A comparative study on illumination preprocessing in face recognition. Pattern Recognition, 46(6):1691-1699, 2013. 

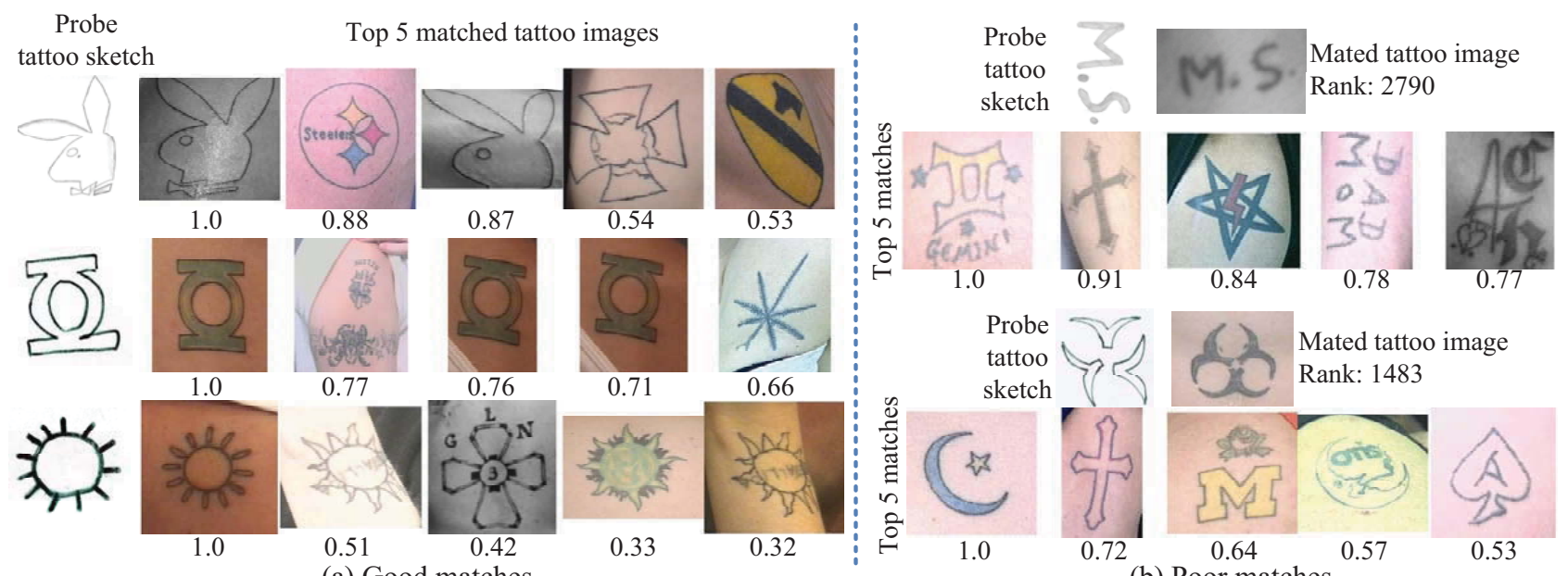

(a) Good matches

(b) Poor matches

Figure 14. Examples of tattoo sketch to image matching. (a) Good matches where rank-1 retrieval is the correct match, (b) Poor matches where the mated tattoo images are matched beyond rank-1000 retrievals. The number under each tattoo image is the match score in the range $[0,1]$.

[12] H. Han, S. Shan, X. Chen, S. Lao, and W. Gao. Separability oriented preprocessing for illumination-insensitive face recognition. In Proc. ECCV, pages 307-320, 2012.

[13] B. Heflin, W. J. Scheirer, and T. E. Boult. Detecting and classifying scars, marks, and tattoos found in the wild. In Proc. BTAS, 2012.

[14] A. K. Jain, S. C. Dass, and K. Nandakumar. Soft biometric traits for personal recognition systems. In Proc. ICBA, pages 731-738, 2004.

[15] A. K. Jain, B. Klare, and U. Park. Face matching and retrieval in forensics applications. IEEE Multimedia, 19(1):20-28, 2012.

[16] A. K. Jain, J. Lee, and R. Jin. Tattoo-ID: Automatic tattoo image retrieval for suspect and victim identification. In Proc. IEEE PCM, pages 256-265, 2007.

[17] Y. Ke, R. Sukthankar, and L. Huston. An efficient partsbased near-duplicate and sub-image retrieval system. In Proc. ACM Multimedia, pages 869-876, 2004.

[18] B. Klare, Z. Li, and A. K. Jain. Matching forensic sketches to mug shot photos. IEEE Trans. Pattern Anal. Mach. Intell., 33(3):639-646, 2011.

[19] J. Lee, R. Jin, and A. K. Jain. Unsupervised ensemble ranking: Application to large-scale image retrieval. In Proc. $I C P R$, pages 3902-3096, 2010.

[20] J. Lee, R. Jin, and A. K. Jain. Image retrieval in forensics: Tattoo image database application. IEEE MultiMedia, 19(1):40-49, 2012.

[21] S. Liao, A. K. Jain, and S. Z. Li. Partial face recognition: Alignment free approach. IEEE Trans. Pattern Anal. Mach. Intell., 2012 (To Appear).

[22] D. G. Lowe. Distinctive image features from scale-invariant keypoints. Int. J. Comput. Vision, 60(2):91-110, 2004.
[23] K. Mikolajczyk and C. Schmid. Scale \& affine invariant interest point detectors. Int. J. Comput. Vision, 60(1):63-86, 2004.

[24] D. Mumford, S. M. Kosslyn, L. A. Hillger, and R. J. Hernstein. Discriminating figure from ground: The role of edge detection and region growing. Proc. Nat. Acad. Sci. USA, 84:7354-7358, 1987.

[25] New York State Division of Criminal Justice Services. Origins of the New York state bureau of identification. 1997.

[26] M. Sharifi, M. Fathy, and M. T. Mahmoudi. A classified and comparative study of edge detection algorithms. In Proc. IEEE ITCC, pages 117-120, 2002.

[27] X. Tang and X. Wang. Face sketch recognition. IEEE Trans. Cir. and Sys. Vid. Tech., 14(1):50-57, 2004.

[28] The US Federal Bureau of Investigation. Next Generation Identification. http://www.fbi.gov/about-us/ cjis/fingerprints_biometrics/ngi.

[29] T. Tuytelaars and L. V. Gool. Content-based image retrieval based on local affinely invariant regions. In Proc. Int'l Conf. VIS, pages 493-500, 1999.

[30] X. Wang and X. Tang. Face photo-sketch synthesis and recognition. IEEE Trans. Pattern Anal. Mach. Intell., 31(11):1955-1967, 2009.

[31] J. Wright, A. Y. Yang, A. Ganesh, S. S. Sastry, and Y. Ma. Robust face recognition via sparse representation. IEEE Trans. Pattern Anal. Mach. Intell., 31(2):210-227, 2009.

[32] P. C. Yuen and C. H. Man. Human face image searching system using sketches. IEEE Trans. Sys. Man and Cyber. Part A, 37(4):493-504, 2007.

[33] W. Zhang, X. Wang, and X. Tang. Coupled informationtheoretic encoding for face photo-sketch recognition. In Proc. IEEE CVPR, pages 513-520, 2011. 